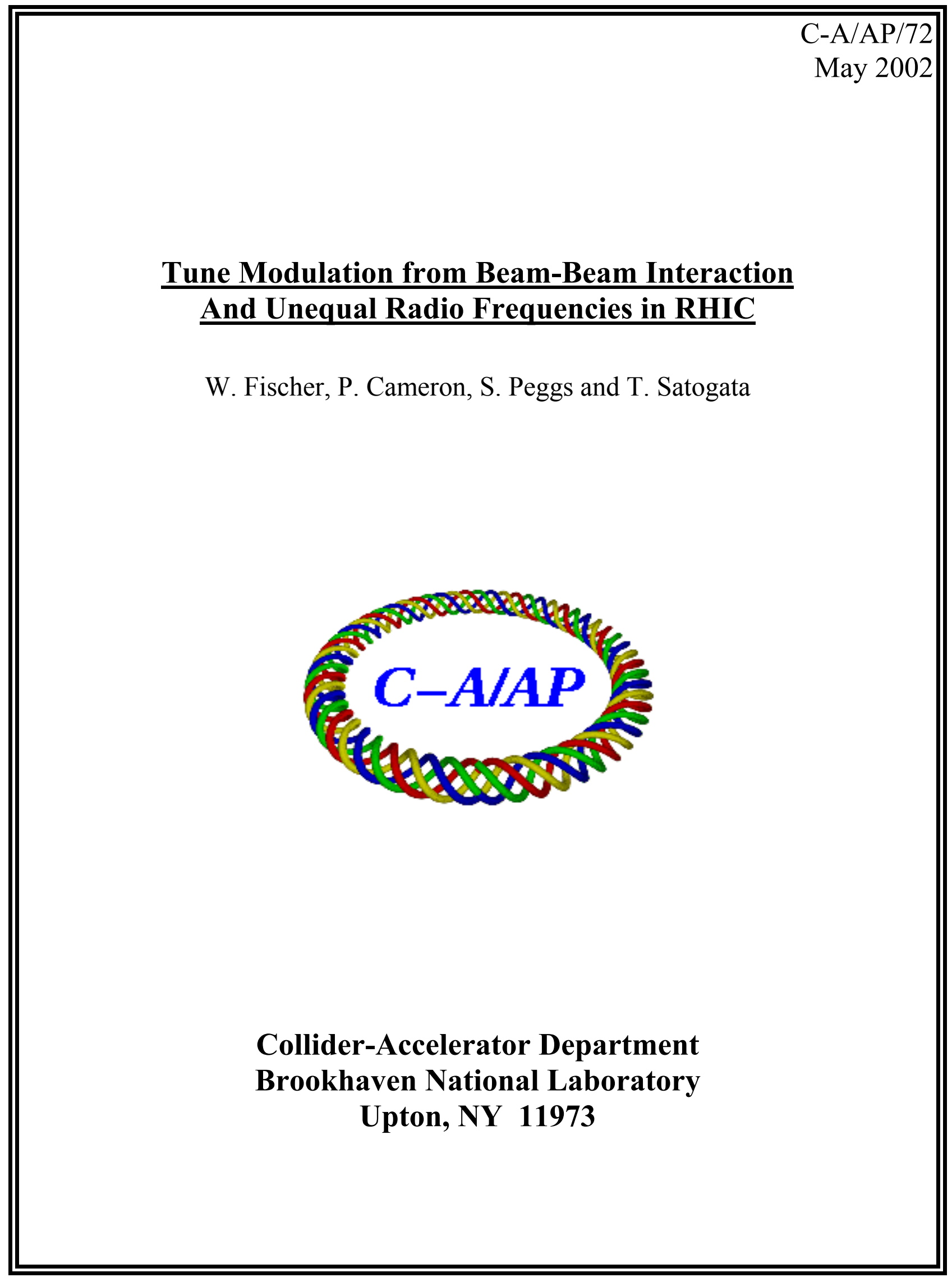




\title{
Tune Modulation from Beam-Beam Interaction and Unequal Radio Frequencies in RHIC
}

\author{
W. Fischer, P. Cameron, S. Peggs and T. Satogata
}

May 18, 2002

\begin{abstract}
The two RHIC rings have independent rf systems to accommodate different species. Thus, the radio frequencies can differ when the phase and radial loops are closed, and the rf frequencies of the two rings are not synchronized. A radio frequency difference leads to longitudinally moving beam crossing points. When the crossing points are between the beam splitting dipoles, the beams experience the beam-beam interaction. Outside the interaction region the beam-beam interaction is switched off. In this way the tune is modulated. A computation of the tune modulation depth, pulse shape and frequency is presented. Tune modulation measurements are shown.
\end{abstract}

\section{Introduction}

During the early RHIC operation it has been observed that beams with 56 bunches suffer lifetime degradations as soon as the rf phase and radial loops are closed at injection. In Fig. 1 an extreme case for such an observation is shown. Fig. 2 shows the same effect at storage energy when the radio frequencies are temporarily unlocked to align the abort gaps.

Beams are injected with equal radio frequencies in both rings and longitudinal separation [1]. Before the ramp starts the phase and radial loops are closed, which leads to a small difference in the radio frequencies since both rings have independent rf systems. A typical difference is between zero and $100 \mathrm{~Hz}$, while the radio frequency at injection is $28.022 \mathrm{MHz}$ for gold beams. The difference in the radio frequencies results in a longitudinally moving beam crossing point.

When the beam crossing point moves through the interaction region (IR), one beam will experience the field of the other beam when the crossing point is located between the DX magnets (see Fig. 3). Outside this region the beams are well separated and do not see the fields of the other beam. Thus, the difference in the radio frequencies leads to a tune modulation.

In the 2001/2002 run a total beam-beam tune spread of $\Delta Q_{b b}=0.005$ was achieved in gold operation with 4 collisions. In proton operation the tune spread reached $\Delta Q_{b b}=0.01$. This tune spread corresponds to the tune modulation depth of small amplitude particles. In the following we compute the tune modulation frequency and wave form taking into account crossing angles. Tune modulation measurements with the phase locked loop (PLL) are presented. 


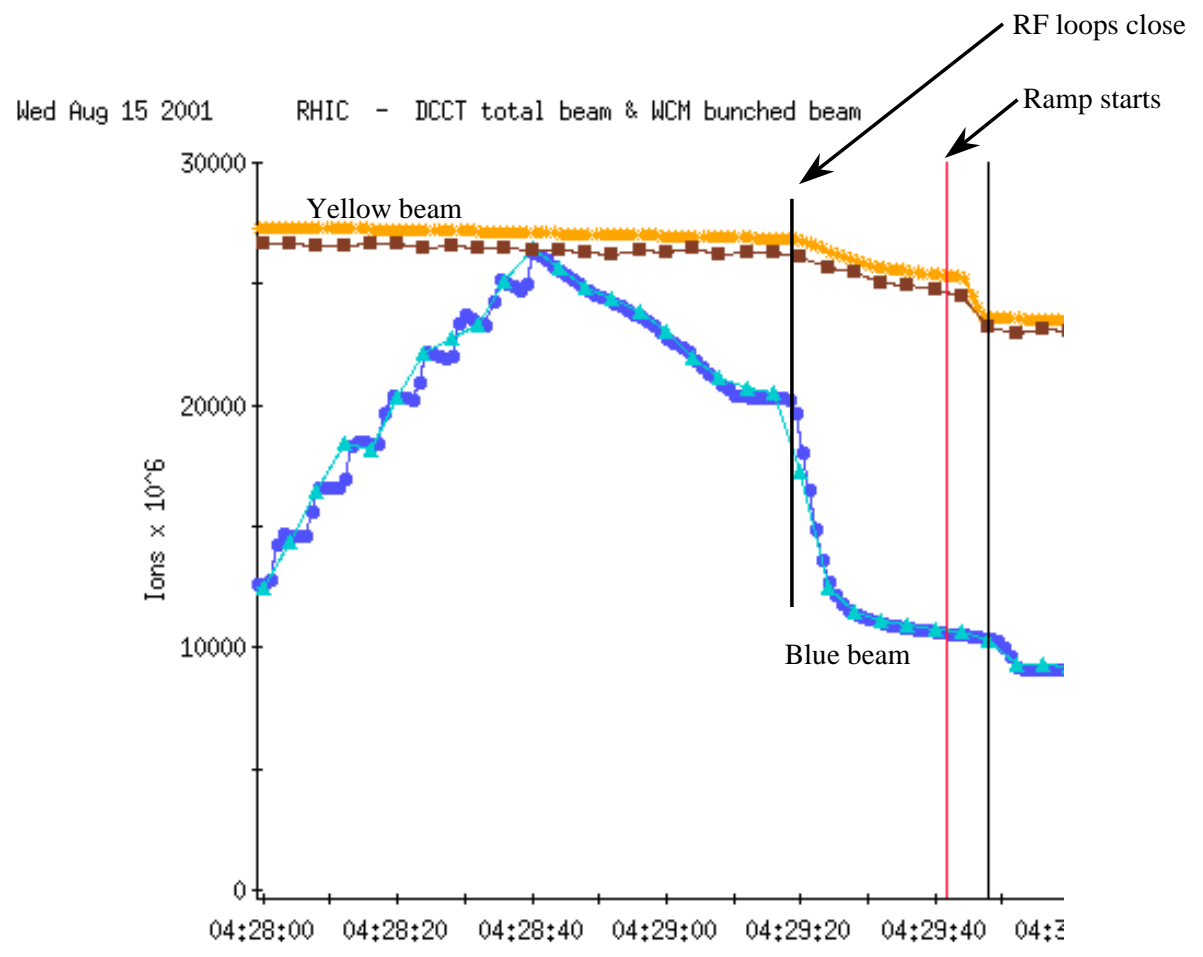

Figure 1: Blue and Yellow lifetime deterioration due to beam-beam interaction with unequal radio frequencies.

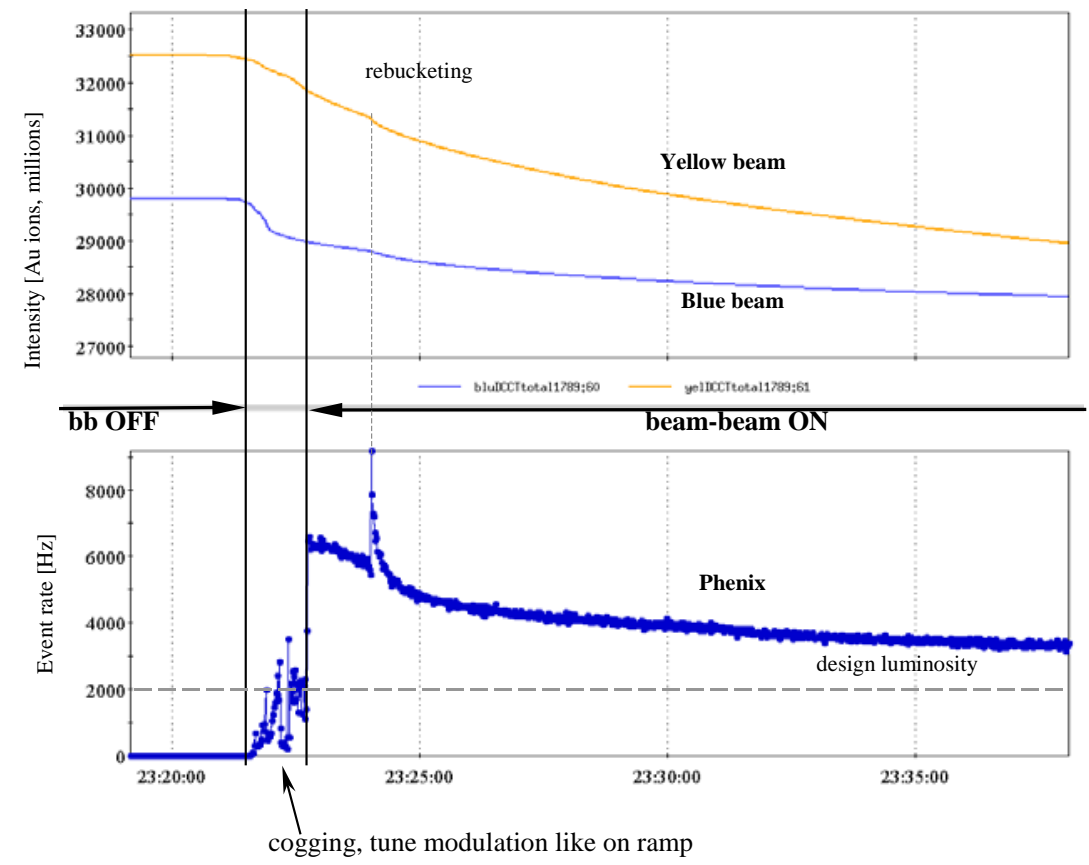

Figure 2: Blue and Yellow lifetime deterioration during cogging at storage energy. 


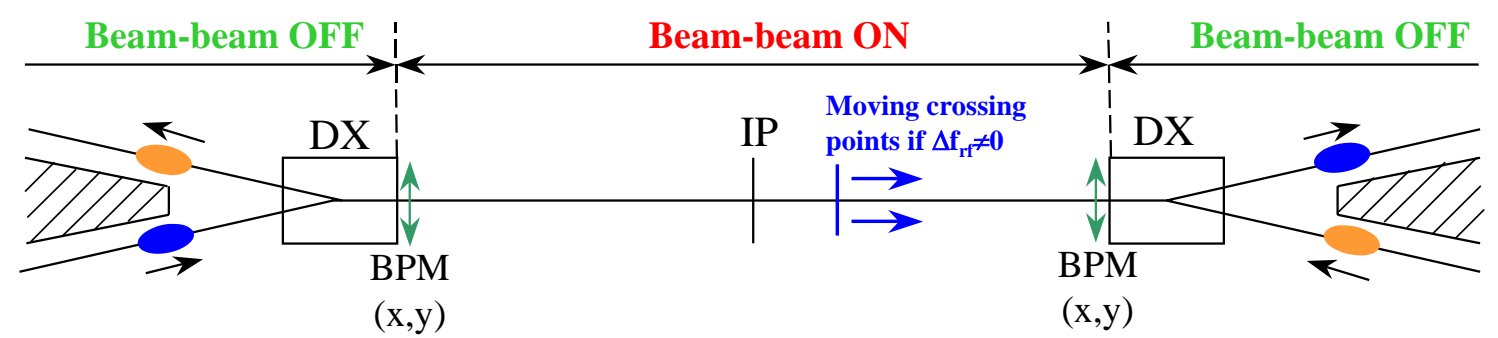

Figure 3: Schematic of RHIC interaction regions layout indicating region where the beam-beam interaction is on and off.

\section{Tune Modulation Parameters}

Denoting the two radio frequencies with $f_{r f, 1}$ and $f_{r f, 2}$ and the harmonic number with $h$, the difference in the revolution time of the two rings is

$$
\Delta T=T_{1}-T_{2}=\frac{h}{f_{r f, 1}}-\frac{h}{f_{r f, 2}}=\frac{h \Delta f_{r f}}{f_{r f}^{2}} .
$$

The crossing point moves by $c \Delta T / 2$ per turn, where $c$ is the particle velocity. The velocity of the crossing point can then be computed as

$$
v_{C P}=\frac{c}{2} \frac{\Delta f_{r f}}{f_{r f}} .
$$

Example RHIC: With $c=2.998 \cdot 10^{8} \mathrm{~ms}^{-1}, f_{r f}=28.022 \mathrm{MHz}$ and $\Delta f_{r f}=5 \mathrm{~Hz}$, it follows $v_{C P}=27 \mathrm{~ms}^{-1}$.

We now compute the wave form of the tune modulation as the crossing point moves from one crotch to the crotch of the other side of the interaction region. Assuming round beams and head-on collisions, particles with small betatron amplitudes will experience horizontal and vertical tune shifts $\Delta Q_{b b}$ between the DX magnets. The tune shifts will become negligible when the crotches are approached. If $L$ is the distance between the crotches, the crossing point moves in time

$$
T_{I R}=\frac{L}{v_{C P}}=\frac{2 L}{c} \frac{f_{r f}}{\Delta f_{r f}}
$$

from one crotch to the other. Particles with larger betatron amplitudes show a smaller tune modulation depth.

Example RHIC: With the values in the above example and $L=31.4 \mathrm{~m}$, it follows $T_{I R}=1.16 \mathrm{~s}$.

The tune shift, rms beam size and beam separation as a function of the longitudinal position are shown in Fig. 4 assuming no crossing angle, and in Fig. 5 assuming a half crossing angle of $0.22 \mathrm{mrad}$. Without a crossing angle the beam-beam interaction is effectively switched off when the crossing point reaches the DX magnets. 


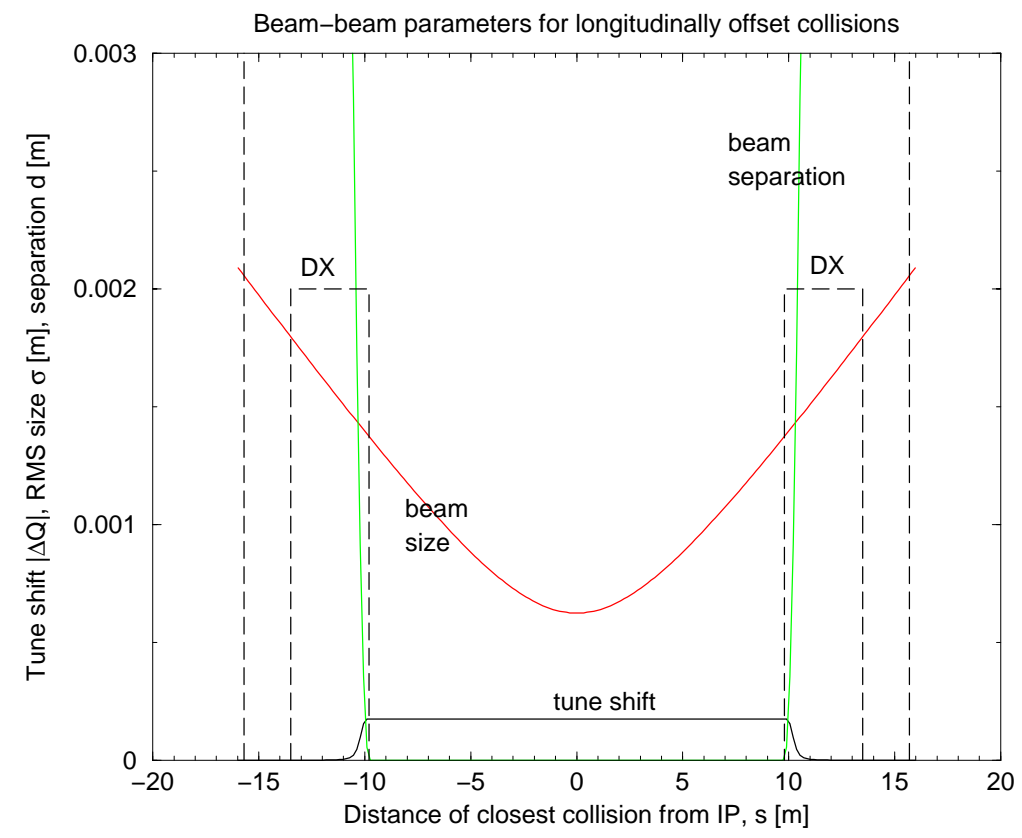

Figure 4: The tune shift (black), rms beam size (red) and beam separation (green) as a function of the longitudinal position without a crossing angle. Assumed are one crossing point, $\xi=\Delta Q_{b b}=0.0002$, $\beta^{*}=5 \mathrm{~m}, \epsilon_{N, 95 \%}=50 \mu \mathrm{m}$. The objects starting at $10 \mathrm{~m}$ from the IP are the DX magnets, the crotches are at $16 \mathrm{~m}$ from the IP.

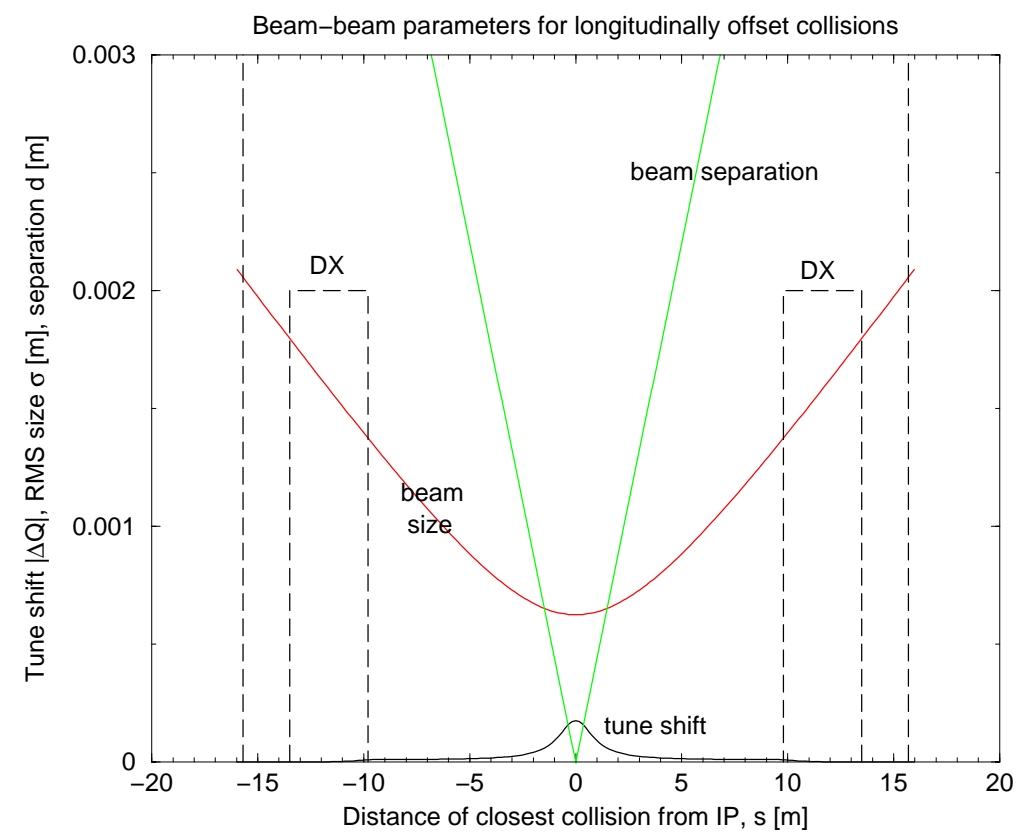

Figure 5: The tune shift (black), rms beam size (red) and beam separation (green) with the same parameters as in Fig. 4, except for the a half crossing angle of $0.22 \mathrm{mrad}$. 
With a crossing angle the beam-beam interaction can be switched off much earlier. With $0.22 \mathrm{mrad}$ half crossing angle (see Fig. 5) the beam-beam interaction is switched off after only a few meters. In 2001 the Brahms experiments was running with a half crossing angle of up to 0.9 mrad due to concerns about the quench performance of an DX magnet in this interaction region.

How often the tune modulation waveform computed above is executed depends on the fill pattern. We assume here rings that are symmetrically filled with $N$ bunches. The distance between IPs is then $C /(2 N)$, where $C$ is the circumference. The time between two waveform executions is then

$$
T_{C}=\frac{C}{2 N v_{C P}}=\frac{C}{N c} \frac{f_{r f}}{\Delta f_{r f}} .
$$

Example RHIC: With the values in the above examples and $N=6$, it follows $T_{C}=11.9$ s. With $N=60$ it follows $T_{C}=1.19 \mathrm{~s}$.

In Fig. 6 the tune modulation wave form is shown schematically. Figs. 7 and 8 show the situation for RHIC with 6 and 60 bunches respectively. With 60 bunches and the numbers in the examples, one has a tune modulation with the frequency $f_{\text {mod }}=0.9 \mathrm{~Hz}$ and a modulation depth of up to a few $10^{-3}$. The modulation depth can thus reach values that are orders of magnitudes larger than modulation depth from power supply ripple. Since the wave form is not sinusoidal, higher tune modulation harmonics are also created.

The effect of tune modulation on the dynamic aperture has been studied with experiments and simulations for the Tevatron [3,4], SPS [5], HERA [6-8], RHIC [9] and the LHC $[10,11]$. A detrimental effect of tune modulation on the dynamic aperture and lifetime was found even with tune modulation depth well below $10^{-3}$.

To avoid the reduction in beam lifetime and dynamic aperture, the RHIC beams were separated vertically by $10 \mathrm{~mm}$ during injection and on the ramp [12]. Furthermore, a scheme was tested at injection to synchronize the two beams during the ramp [13].

\section{Tune Modulation Measurements}

In figures 9, 10 and 11 measurements are shown that illustrate tune modulation mechanism. All measurements were done with a phase locked loop (PLL), which allows to measure the tune to better than $10^{-4}$.

In Fig. 9 the tune shift is measured when the bunches are separated longitudinally, i.e. there crossing point is beyond the DX magnet. The crossing point is then moved in 3 steps until it is at the nominal interaction point. A cogging step by one bucket moves the crossing point by $5 \mathrm{~m}$ longitudinally. The tune shift corresponding to the last crossing point is a sign of crossing angles (compare with Fig. 5). With beams colliding the tune is depressed through the beam-beam interaction. From the measured beam-beam tune shift the emittance can be inferred since the bunch currents are known. 


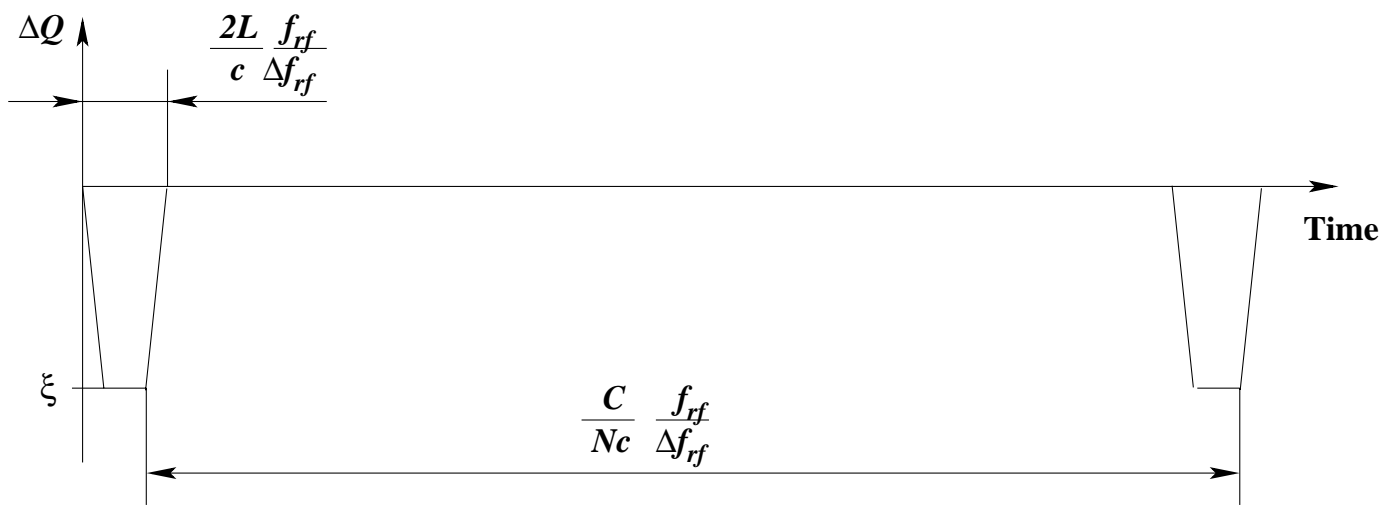

Figure 6: Tune modulation due to beam-beam interaction with unequal radio frequencies. Shown are the length of the beam-beam interaction and the time between beam-beam interactions.

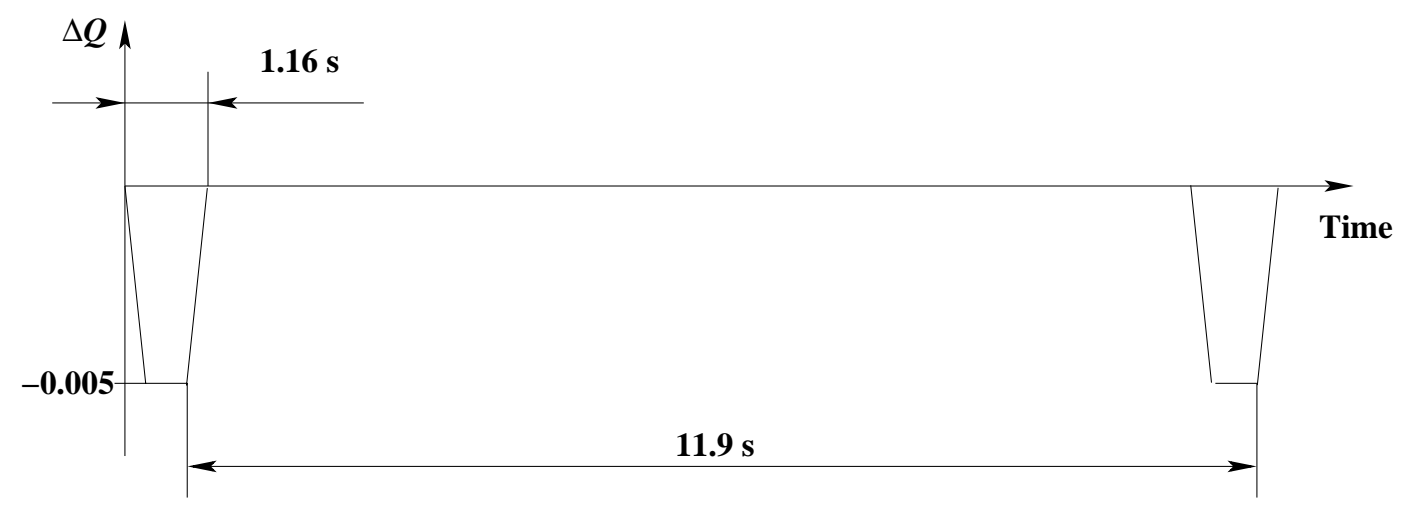

Figure 7: Tune modulation with parameters as in the RHIC examples and 6 symmetrically distributed bunches in each ring.

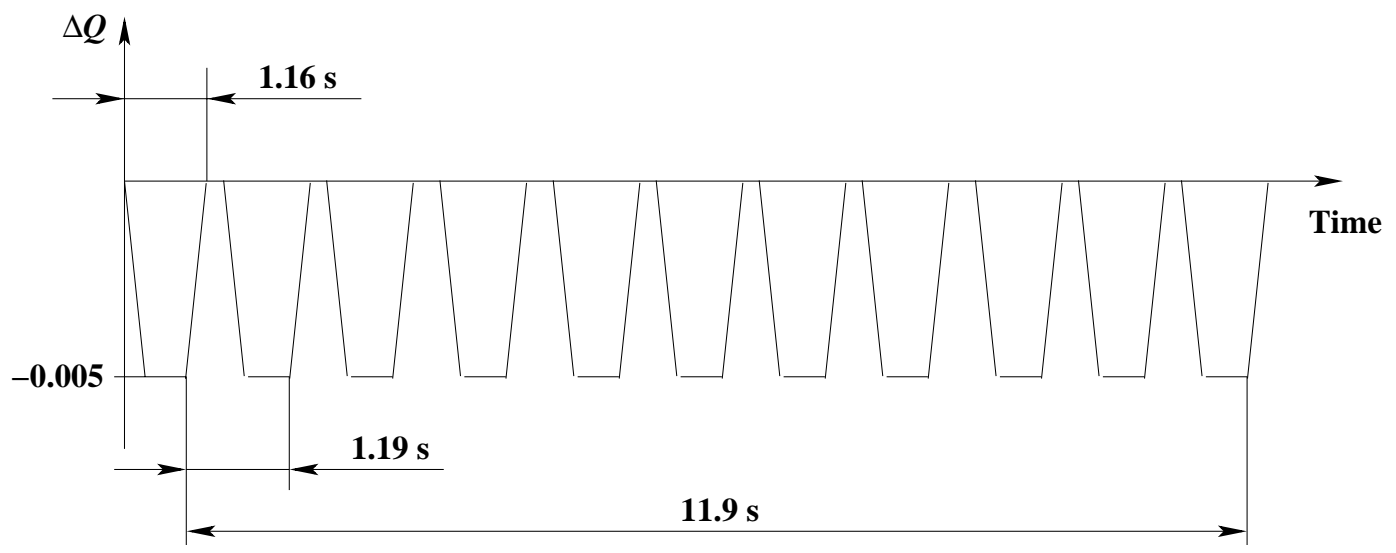

Figure 8: Tune modulation with parameters as in the RHIC examples and 60 symmetrically distributed bunches in each ring. 


\section{PLL Blue horizontal,}

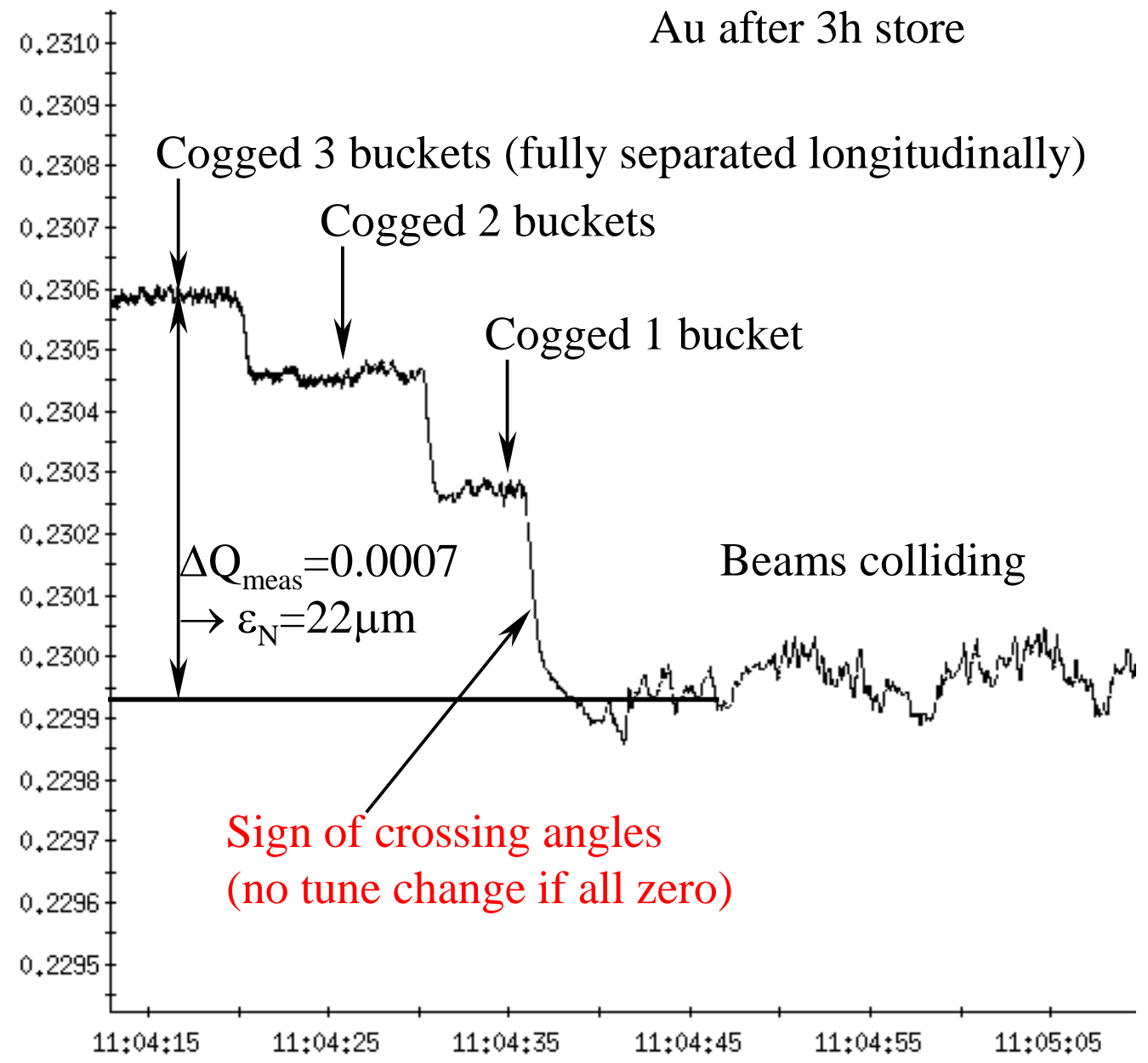

Figure 9: Tune shift due to beam-beam interaction with different longitudinal locations of the beam crossing points. The measurement was taken after gold beam was stored for three hours.

For Gaussian round beams the beam-beam parameter is

$$
\xi=\frac{3 N_{b} r}{2 \pi \epsilon_{N}}
$$

where $N_{b}$ is the bunch intensity, $r$ the classical particle radius, and $\epsilon_{N}$ the normalized 95\% emittance. The observed tune shift is approximately

$$
\Delta Q=\frac{1}{2} N_{x} \xi
$$

where $N_{x}$ is the number of beam-beam interactions per turn. The factor $1 / 2$ stems from the the observation of the coherent tune shift [14]. 


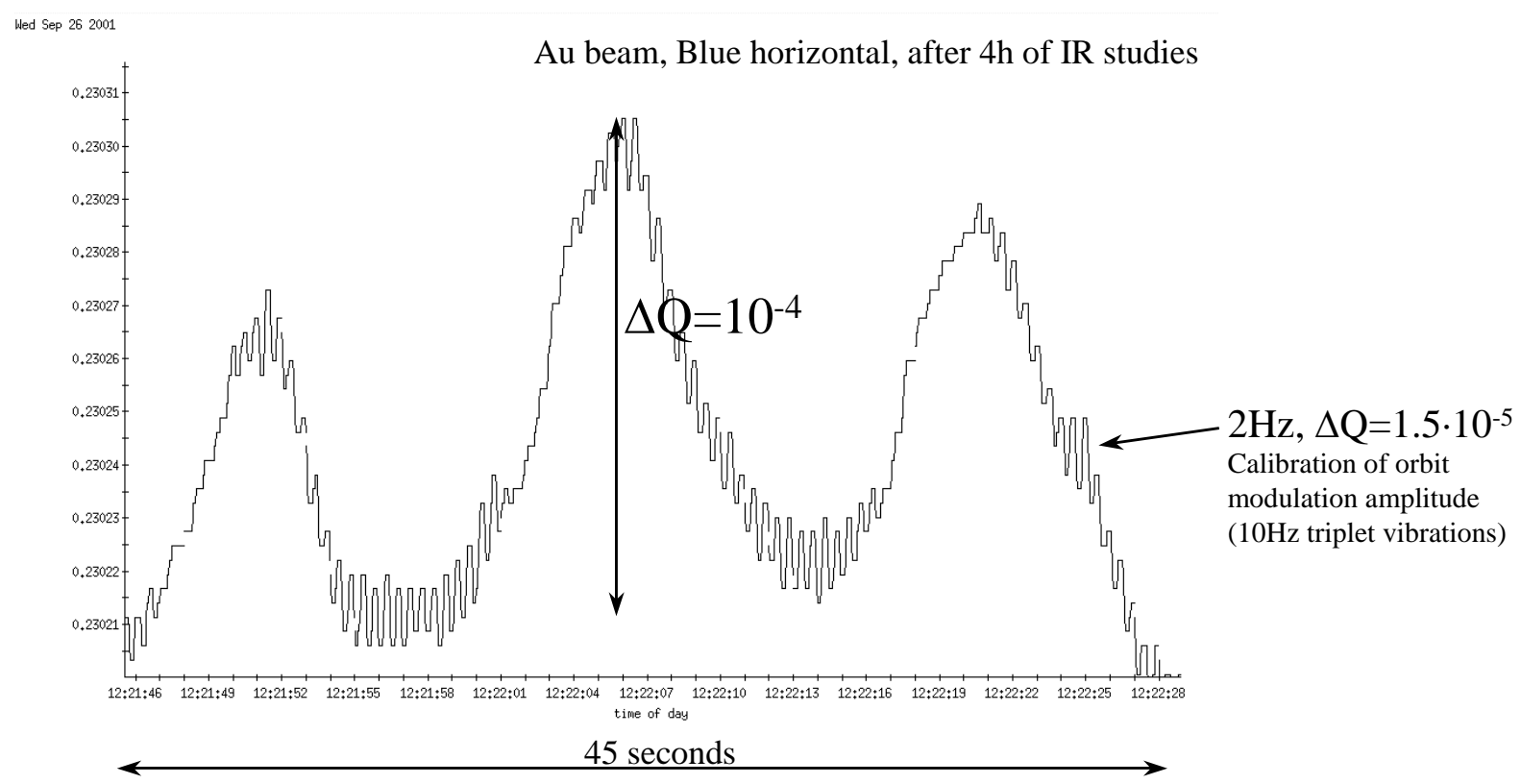

Figure 10: Tune modulation with unlocked radio frequencies at storage energy. In both rings are 55 gold bunches.

In Fig. 10 a situation is shown where the radio frequencies at store were unlocked to calibrate a $10 \mathrm{~Hz}$ orbit modulation line with a $2 \mathrm{~Hz}$ radius modulation. The unlocked radio frequencies lead to a tune modulation due to the beam-beam interaction.

In Fig. 11 the Blue vertical tune and the Yellow horizontal tune are shown at the end of the ramp and during rf manipulations at flat top. In each ring there are 55 bunches. In the Blue ring there are, on average, $6.36 \cdot 10^{10}$ protons per bunch, in the Yellow ring there are $4.27 \cdot 10^{10}$ protons per bunch. During the energy ramp both tunes are moving. In addition, the tunes are modulated since the $\mathrm{rf}$ frequencies are unlocked. There is a modulation period of $T_{c}=3.3 \mathrm{~s}$ during the ramp and, according to Eq. (4), the difference between the radio frequencies is $\Delta f_{r f}=1.8 \mathrm{~Hz}$. The rf frequency is $f_{r f}=28.149 \mathrm{MHZ}$. When reaching the flat top, the tune modulation stops when the rf frequencies are synchronized. Then the abort gaps are aligned longitudinally ("cogging"). During this process the rf frequency of one ring is slightly changed temporarily to allow one bunch pattern to move against the other one. The maximum frequency difference in the process is $\Delta f_{r f}=10 \mathrm{~Hz}$. This leads to a modulation period of $T_{c}=0.6 \mathrm{~s}$ that is also measured with the PLL. When the cogging is finished the tunes are depressed by the beam-beam interaction. The Blue beam probes the Yellow beam and thus its tune depression depends on the Yellow emittance and bunch intensity. The Yellow beam probes the Blue beam. If both beams have the same emittance, it follows from Eq. (5)

$$
\frac{\Delta Q_{\text {Blue }}}{\Delta Q_{\text {Yellow }}}=\frac{N_{b, \text { Yellow }}}{N_{b, \text { Blue }}}
$$

in accordance with the observation shown in Fig. 11. 


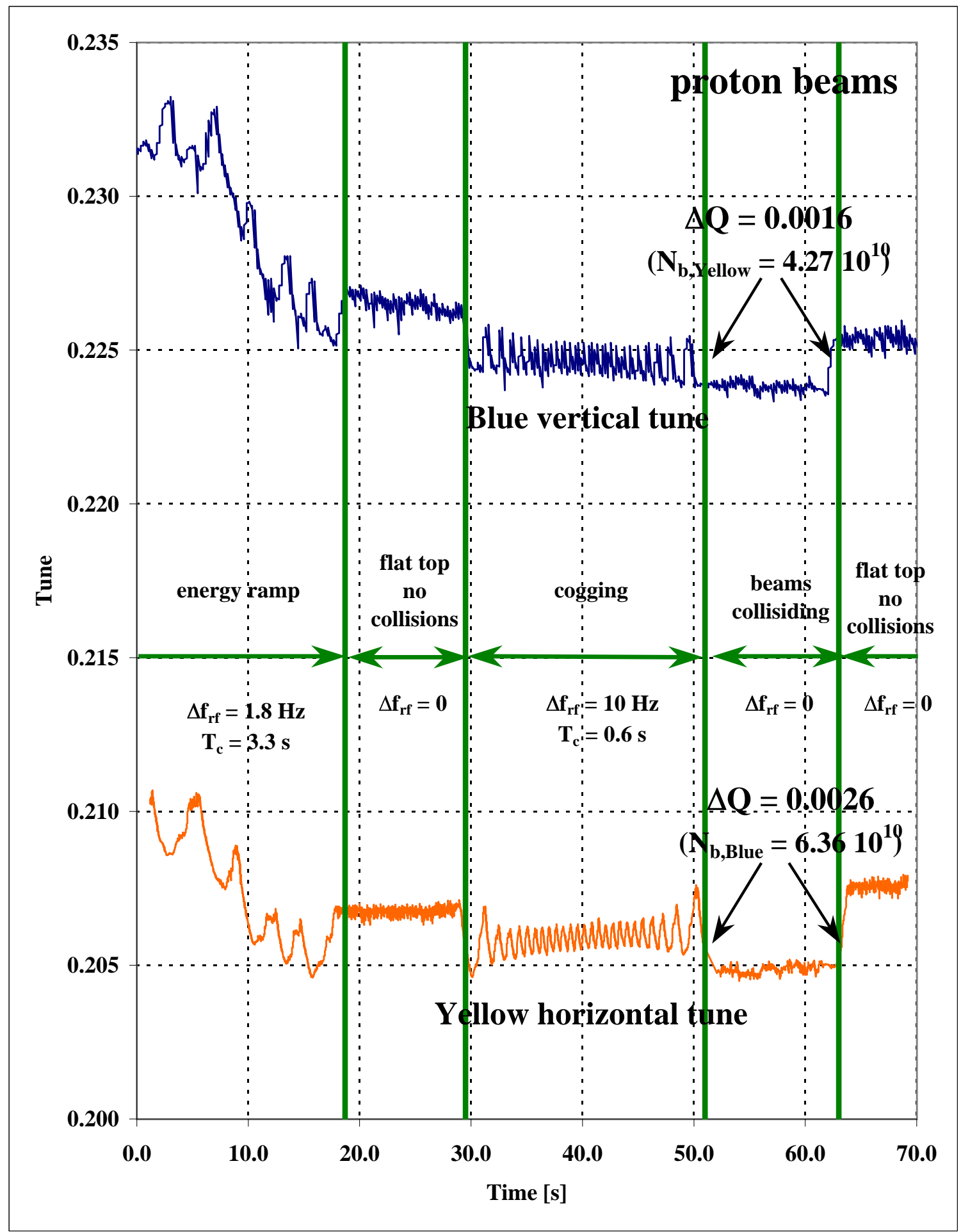

Figure 11: Blue vertical and Yellow horizontal tune, measured with the PLL, at the end of the ramp and during rf manipulations at flat top. 


\section{Summary}

When the two RHIC beams have slightly different radio frequencies the tune is modulated with frequencies of typically 5 to $100 \mathrm{~Hz}$ with modulation depths of up to a few $10^{-3}$, the total beam-beam tune shift. Measurements and simulations for existing hadron machines have shown that such conditions are likely to affect the dynamic aperture and beam lifetime severely. This has also been observed in RHIC.

\section{Acknowledgements}

The authors would like to thank for help and discussions: M. Bai, M. Blaskiewicz, J.M. Brennan, J. DeLong, T. Hayes, V. Ptitsyn, S. Tepikian, T. Roser, and D. Trbojevic.

\section{References}

[1] J. DeLong, J.M. Brennan, private communication (2001).

[2] W. Fischer and S. Peggs, "RHIC as a Test Bench for Beam-Beam Studies", proceedings of the Beam-Beam Workshop, Fermilab (2001).

[3] A. Chao et al., "Experimental investigations on nonlinear dynamics in the Fermilab Tevatron", Phys. Rev. Lett. 61, pp. 2752-2755 (1988).

[4] T. Satogata, "Nonlinear resonance islands and modulational effects in a proton synchrotron", PhD Thesis, Northwestern University (1993).

[5] W. Fischer, M. Giovannozzi and F. Schmidt, "Dynamic Aperture Experiment at a Synchrotron", Phys. Rev. E, Vol. 55, Number 3, p 3507 (1997).

[6] F. Zimmermann, "Emittance Growth and Proton Beam Lifetime in HERA", PhD thesis, Hamburg University, DESY 93-059 (1993).

[7] O. Brüning, "An Analysis of the Long-term Stability of the Particle Dynamics in Hadron Storage Rings", PhD thesis, Hamburg University, DESY 94-085 (1994).

[8] W. Fischer, "An Experimental Study on the Long-term Stability of Particle Motion in Hadron Storage Rings", PhD thesis, Hamburg University, DESY 95-235 (1995).

[9] W. Fischer and T. Satogata, "A Simulation Study On Tune Modulation Effects in RHIC", BNL RHIC/AP/109 (1996).

[10] W. Fischer and F. Schmidt, "Long-term Tracking for the LHC including ripple", CERN SL/Note 94-75 (1994).

[11] M. Böge, A. Faus-Golfe, J. Gareyte, H. Grote, J.-P. Koutchouk, J. Miles, Q.Qing, T. Risselada, F. Schmidt, S. Weisz, "Overview of the LHC dynamic aperture studies", proceedings of the 1997 Particle Accelerator Conference, Vancuver (1997).

[12] V. Ptitsyn, private communication (2001).

[13] J.M. Brennan, private communication (2001).

[14] E. Keil, "Beam-beam Dynamics", CERN Accelerator School, Rhodes, Greece, CERN 95-06 (1995), CERN SL/94-78 (1994). 